Note

\section{Breeding of a Sake Yeast with Improved Ethyl Caproate Productivity}

\author{
Eiji IcHIKawa, Naomi HosoKawa, \\ Yoji Hata, Yasuhisa ABE, \\ Koji Suginami and Satoshi Imayasu
}

\section{Research Institute, Gekkeikan Sake Co., Ltd., 24, Shimotoba Koyanagi-cho, Fushimi-ku, Kyoto 612, Japan}

Received December 25, 1990

Flavor components, especially isoamyl acetate and ethyl caproate, are important for the quality of sake. A special technique called ginjozukuri, which uses highly polished rice and low temperature fermentation, is usually used to brew sake rich in these esters. It is not easy, even with this technique, to brew rich-flavored sake. It is therefore important to breed a yeast strain producing flavor components at high levels in the sake mash. We have isolated a mutant producing sufficient isoamyl acetate by selecting 5,5,5-trifluoroleucine-resistant mutants. ${ }^{1 \text { ' }}$

The synthesis of ethyl caproate in sake mash was catalyzed by enzymes in yeast; esterase and alcohol acyltransferase. ${ }^{2)}$ The substrates of these enzymes for the synthesis of ethyl caproate are ethanol and caproic acid or caproyl-CoA. As there is sufficient ethanol in th sake mash, either caproic acid or caproyl-CoA is a rate-limiting factor for the synthesis. The production of caproic acid is important in producing sake with a large amount of ethyl caproate. In this study we isolated mutant yeasts that produce sufficient caproic acid and ethyl caproate from sake yeast as strains resistant to cerulenin, a specific inhibitor of fatty acid synthetase. ${ }^{3)}$

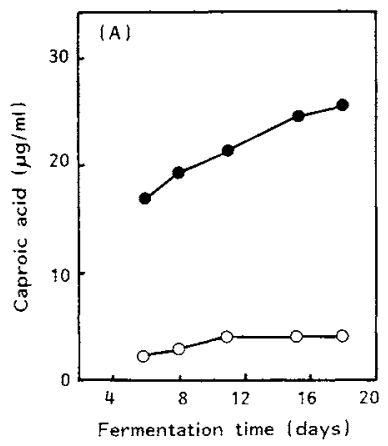

The pathway of caproic acid synthesis is not known in sake yeast. It has been reported, however, that medium-chain fatty acids in beer are produced by fatty acid synthesis, and not introduced by degradation of long chain fatty acids. ${ }^{4}$ Although detailed data were not shown, we have been suggested that caproic acid synthesis by sake yeast is inhibited by cerulenin. From these results, we assumed that caproic acid is produced by fatty acid synthesis in sake yeast. Fatty acid synthesis of the yeast is catalyzed by fatty acid synthetase, a multifunctional enzyme with seven different catalytic activities. ${ }^{5)}$ Alteration in balance of the individual catalytic activities in fatty acid synthetase changes the chain length of synthesized fatty acids. ${ }^{6}$ Since cerulenin is a inhibitor of fatty acid synthetase, it was expected that caproic acid productivity of a cerulenin-resistant mutant would be different from that of the wild strain.

Haploid yeast (G1103), isolated from the sake yeast (Saccharomyces cerevisiae) Kyokai No. 7, was mutagenized by ethylmethane sulfonate as described previously ${ }^{13}$ and spread on YEPD agar plates ( $1 \%$ yeast extract, $2 \%$ peptone, $2 \%$ glucose, $2 \%$ agar) containing $25 \mu \mathrm{m}$ cerulenin at $30^{\circ} \mathrm{C}$. Fifty-four colonies that developed on the plates were isolated as mutants resistant to cerulenin. The mutants were inoculated into the medium containing $0.67 \%$ Difco yeast nitrogen base without amino acid, $1 \%$ casamino acid, and $5 \%$ glucose, and the cultures were incubated for 3 days at $30^{\circ} \mathrm{C}$. Then the broth was centrifuged and the resultant supernatant was used for analysis of caproic acid. Samples of the supernatant $(100 \mathrm{ml})$ were adjusted to $\mathrm{pH}$ 1.0 with $\mathrm{HCl}$ and total lipids were extracted once with $100 \mathrm{ml}$ of ethyl ether. The extract was washed once with diluted $\mathrm{HCl}$ and dried over anhydrous $\mathrm{Na}_{2} \mathrm{SO}_{4}$. After this was concentrated with a Kderna-Danish apparatus, the sample was adjusted to $1.0 \mathrm{ml}$ with ethyl ether and analyzed by gas chromatography under the following conditions; column, $10 \%$ FON on Celite 545; column temperature, $200^{\circ} \mathrm{C}$; nitrogen flow rate $50 \mathrm{ml} / \mathrm{min}$. Among fifty-four mutants, three produced more than three times as much caproic acid as G1103 did, while the other mutants produced the same level of caproic acid as G1103

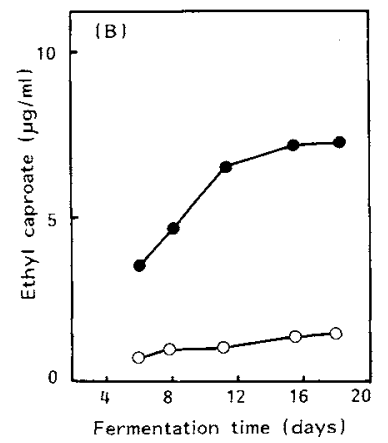

Fig. 1. Changes in Caproic Acid and Ethyl Caproate Concentrations in Sake Mash. (A), caproic acid; (B), ethyl caproate; O, G1103; O, C-8. 
Table I. Components of Sake Fermented WITH G1103 AND C-8

\begin{tabular}{|c|c|c|}
\hline Component & G1103 & $\mathrm{C}-8$ \\
\hline Sake meter ${ }^{a}$ & -1.0 & -3.5 \\
\hline AlcohoI $(\%)^{a}$ & 17.4 & 17.1 \\
\hline Acidity $(\mathrm{ml})^{a}$ & 2.72 & 2.93 \\
\hline Amino acidity $(\mathrm{mI})^{a}$ & 2.50 & 2.72 \\
\hline Isobutyl alcohol $(\mu \mathrm{g} / \mathrm{ml})$ & 110 & 102 \\
\hline Isoamyl alcohol $(\mu \mathrm{g} / \mathrm{ml})$ & 257 & 207 \\
\hline Isoamyl acetate $(\mu \mathrm{g} / \mathrm{ml})$ & 6.8 & 5.7 \\
\hline Ethyl caproate $(\mu \mathrm{g} / \mathrm{ml})$ & 1.4 & 7.4 \\
\hline Ethyl caprylate $(\mu \mathrm{g} / \mathrm{ml})$ & 0.5 & 1.5 \\
\hline
\end{tabular}

a Measured by the method of the National Research Institute of Brewing (Tax Administration Agency, Japan).

Table II. Organic Acid Composition of SAKE WITH G1103 AND C-8

\begin{tabular}{lcc} 
& \multicolumn{1}{c}{ Concentration $(\mu \mathrm{g} / \mathrm{ml})$} \\
\cline { 2 - 3 } Organic acid & G1103 & C-8 \\
\hline Lactic acid & 452 & 423 \\
Acetic acid & 22 & 18 \\
Citric acid & 52 & 60 \\
Succinic acid & 243 & 216 \\
Malic acid & 427 & 463 \\
Caproic acid & 4.4 & 24.9 \\
Caprylic acid & 1.7 & 4.9 \\
Capric acid & 0.7 & 1.7
\end{tabular}

accumulated, C-8 strain, which produced the highest amount of caproic acid, was used for further tests.

Fermentation tests with G1103 and C-8 were done as described previously. ${ }^{11}$ A regular grade of rice (Nipponbare) was used with the polishing ratio of $72 \%$. Sake mash was composed of $1040 \mathrm{~g}$ of steamed rice, $240 \mathrm{~g}$ of koji, and $1400 \mathrm{ml}$ of water, and fermented at a constant temperature of $15^{\circ} \mathrm{C}$. The changes in the concentrations of caproic acid and ethyl caproate in sake mash are shown in Fig. 1. The concentration of caproic acid and ethyl caproate in C-8 mash was much higher than that in Gll03 mash. The components of sake are shown in Table I. The sake meter, alcohol concentration, acidity, and amino acidity in sake brewed with $\mathrm{C}-8$ were similar to those in sake brewed with G1103. However, the concentration of ethyl caproate was more than five times as high as that of G1103, and the concentration of ethyl caprylate also increased. Table II shows the components of organic acids. C-8 produced caproic acid about five times as high as Gl 103 and also caprylic acid and capric acid increased. There was no significant differences in other organic acids. In a sensory evaluation, the sake brewed with C- 8 had an excellent fruity flavor.

Hybrid strains between C-8 and a cerulenin-sensitive strain were resistant to cerulenin. This indicated that cerulenin resistance was dominant and that a similar mutant was obtainable from industrial sake yeast. K-9-3 strain, a mutant with improved caproic acid productivity, was also isolated from sake yeast Kyokai No. 9 (K-9) by the method as used for G1103. C-8, K-9-3, and K-9 were used for sake fermentation tests under the same conditions described above except that $1300 \mathrm{ml}$ of water was used. C-8, K-9-3, and $\mathrm{K}-9$ produced $8.1,4.7$, and $1.1 \mu \mathrm{g} / \mathrm{ml}$ of ethyl caproate, respectively; and $17.5 \%, 18.5 \%$, and $18.7 \%$ alcohol, respectively. K-9-3 produced four times as much ethyl caproate as K-9, and sake brewed with K-9-3 had a superior flavor in the sensory evaluation. Taking alcohol tolerance and fermentation ability into consideration, K-9-3 seems more suitable for sake brewing than C-8.

Although highly polished rice and low temperature fermentation have been required to brew rich-flavored sake, the mutant isolated in this study enabled us to produce sake with sufficient concentration of ethyl caproate by conventional brewing techniques.

\section{References}

1) S. Ashida, E. Ichikawa, K. Suginami and S. Imayasu, Agric. Biol. Chem., 51, 2061 (1987).

2) K. Kuriyama, S. Ashida, Y. Saito, Y. Hata, K. Suginami and S. Imayasu, Hakkokogaku, 64, 253 (1986).

3) S. Omura, Bacteriol. Rev., 40,681 (1976).

4) G. T. Taylor and B. H. Kirsop, J. Inst. Brew., 83, 241 (1977).

5) F. Lynen, Eur. J. Biochem., 112, 431 (1980).

6) M. Sumper, D. Oesterheit, C. Riepertinger and F. Lynen, Eur. J. Biochem., 10, 377 (1969). 